\title{
IEDITORIAL
}

\section{The essential role of a "healthy" relationship between caveolin-1 and endothelial nitric oxide synthase in counteracting vascular inflammation and atherothrombosis}

\author{
Vincenzo Mollace, Annamaria Tavernese, Rocco Mollace \\ 1 Institute of Research for Food Safety \& Health, Department of Health Sciences, University "Magna Graecia" of Catanzaro, Catanzaro, Italy \\ 2 IRCCS San Raffaele Hospital, Rome, Italy
}

RELATED ARTICLE

by Mierke et al, see p. 124
Correspondence to:

Prof. Vincenzo Mollace, MD, PhD, Campus Universitario di Germaneto, University "Magna Graecia" of Catanzaro, 88100 Catanzaro, Italy, email:mollace@unicz.it Received: January 24, 2020. Accepted: January 25, 2020. Published online: February 25, 2020. Kardiol Pol. 2020; 78 (2): 96-97 doi:10.33963/KP.15203

Copyright by the Author(s), 2020
The recent study by Mierke et al, ${ }^{1}$ published in Kardiologia Polska (Kardiol Pol, Polish Heart Journal), highlights the role of caveolin 1 (Cav-1) / constitutive endothelial nitric oxide synthase (eNOS) in adventitial inflammation and, subsequently, in the progression of vascular smooth muscle cell (VSMC) proliferation. This was assessed in a model of the denuded aorta from C57Bl6n (wild-type), $\mathrm{Cav}_{-1}{ }^{-/}$, $\mathrm{eNOS}^{-/}$, as well as Cav-1 ${ }^{-/-}$and eNOS $^{-/-}\left(\mathrm{C}^{-\mathrm{e}^{-/-}}\right)$mice transplanted into common carotid arteries of wild-type mice.

This confirms previous background data which showed that the Cav-1/eNOS system plays a protective role against VSMC proliferation, which is associated with several disease states, including diabetes, hyperlipemia, arterial hypertension, etc, leading to atherosclerotic plaque progression and atherothrombosis. ${ }^{2}$ The activity of Cav-1/ eNOS protective mechanisms is attenuated by inflammatory stimuli associated with an overproduction of inflammatory cytokines which, in turn, reduce eNOS release and, via activation of inducible NOS (iNOS) and cyclooxygenase 2, generate large quantities of $\mathrm{NO}$ and prostanoids. ${ }^{3}$ This effect, alongside oxidative stress, leads to apoptotic cell death of endothelial cells and VSMC proliferation, though the mechanisms still remains to be elucidated.

Evidence clearly showed that a continuous cross-modulation exists between eNOS and iNOS under basal conditions as well as in the development of vascular inflammation. Moreover, it is known that eNOS contributes, under basal conditions, to maintaining nuclear factor $\mathrm{kB}$ (NF-kB) / iNOS not activated. ${ }^{3}$ Recent evidence demonstrates that nM concentrations of NO released by eNOS counteract the binding of NF$-\mathrm{kB}$ to its promoter response element, thereby modulating the activation and translocation of NF-kB. ${ }^{4}$ On the other hand, NO generated by "healthy" eNOS inhibits NF-kB DNA binding through S-nitrosylation of the Cys 62 residue of p50 subunit, thereby maintaining NF-kB under subtoxic concentrations. ${ }^{4}$ The maintenance of this equilibrium between Cav-1/eNOS at one side and NF-kB / iNOS on the opposite side represents the basis for the physiological regulation of vascular functionality. Yet, the disruption of this relationship leads to endothelial dysfunction and vascular inflammation. This is confirmed in the model used by Mierke et al, ${ }^{1}$ obtained by means of Cav-1/ eNOS knockout mice.

Beside the above-mentioned evidence, several aspects on the shift occurring at the level of NF-kB / iNOS system from the inactive "healthy" state into the inflammatory (activated) condition is still to be clarified. However, the involvement of Cav-1 in the regulation of eNOS / iNOS activation states seems to play a relevant role in vascular inflammation.

Compelling evidence shows that caveolae (which are 50-100 $\mathrm{nm}$ vesicular invaginations of the endothelial cell membrane) play a crucial role in vesicular trafficking and modulation of signal transduction including eNOS regulation. ${ }^{5,6}$ This occurs via Cav-1, a 21-to-24-kDa 
protein, which works as a scaffolding protein and negatively modulates phosphorylation of eNOS by interacting with multiple molecules. This effect of Cav-1 on eNOS activation is also regulated by $\mathrm{Ca}^{2+}$-calmodulin. ${ }^{7}$

In particular, it has been shown that the binding of Cav-1 to eNOS is a crucial negative regulator of eNOS activity and that inflammation-related decrease of NO production in endothelial cells is partially due to an increased interaction of Cav-1 and eNOS. ${ }^{8}$ Thus, the mechanism is involved in inflammation-induced endothelial dysfunction and atherosclerosis, though it is still unclear.

Toll-like receptor (TLR) 4 is the major player in the regulation of the activity of Cav- $1 .^{9}$ This receptor has been found to play a crucial role in inflammatory response occurring in vascular and nonvascular tissues. Its function requires Tyr14 phosphorylation of Cav-1 to exert its inflammatory role. ${ }^{10}$ On the other hand, TLR4 starts early recruitment of the adaptor protein MyD88 leading to the activation of IkB kinase, which is involved in phosphorylation and degradation of $\mathrm{IkB}$, leading, in turn, to the release and translocation of NF- $\mathrm{kB}$ to the nucleus. ${ }^{2}$ Thus TLR4, once activated, leads to Cav-1 phosphorylation, thereby inhibiting a constitutive NO release by eNOS. Moreover, TLR4 activates NF$-\mathrm{kB}$, which leads to activation of iNOS, thereby initiating vascular inflammation and VSMC proliferation. This fits very well with data provided by Mierke et al. ${ }^{1}$ Indeed, they found that Cav-1 and eNOS knockout was accompanied by inhibition of vascular endothelial growth factor A expression and VSMC proliferation. It is likely that derepression of iNOS and NF-kB activation, in Cav-1 and eNOS knockout mice may contribute to this response. On the other hand, it is likely that other mechanisms may contribute in the consequences of imbalanced Cav-1/eNOS regulation occurring in vascular inflammation. Indeed, evidence exists that metabolic disorders accompanied by enhanced formation of oxidized low-density lipoprotein lead to the upregulation of Cav-1. ${ }^{2}$ This is associated with an overexpression of the lectin-like oxidized low-density lipoprotein receptor-1, which contributes to translocation of NF-kB with subsequent activation of iNOS and cyclooxygenase 2.

In conclusion, physiological regulation of eNOS and Cav-1 contributes to the maintenance of blood vessels into a noninflammatory state, which is characterized by low tissue concentrations of inducible enzymes, inflammatory mediators, and growth factors. An imbalanced regulation of these mechanisms produces overexpression of TLR4 / NF-kB which, in turn, is accompanied by larger concentrations of inflammatory mediators, mostly from the adventitia, which promote VSMC proliferation and vascular stenosis. Further exploration of these mechanisms should provide useful information for a better management of atherothrombosis.

\section{ARTICLE INFORMATION}

ACKNOWLEDGMENTS This work was supported by the grants of PON-MIUR (no. 00359; to VM, AT, and RM) and the Consortium Nutramed (to VM, AT, and RM). DISCLAIMER The opinions expressed by the author are not necessarily those of the journal editors, Polish Cardiac Society, or publisher.

\section{CONFLICT OF INTEREST None declared.}

OPEN ACCESS This is an Open Access article distributed under the terms of the Creative Commons Attribution-NonCommercial-NoDerivatives $4.0 \mathrm{In}$ ternational License (CC BY-NC-ND 4.0), allowing third parties to download articles and share them with others, provided the original work is properly cited, not changed in any way, distributed under the same license, and used for noncommercial purposes only. For commercial use, please contact the journal office at kardiologiapolska@ptkardio.pl.

HOW TO CITE Mollace V, Tavernese A, Mollace R. The essential role of a "healthy" relationship between caveolin-1 and endothelial nitric oxide synthase in counteracting vascular inflammation and atherothrombosis. Kardiol Pol. 2020; 78: 96-97. doi:10.33963/KP.15203

\section{REFERENCES}

1 Mierke J, Christoph M, Augstein A, et al. Influence of caveolin-1 and endothelial nitric oxide synthase on adventitial inflammation in aortic transplants. Kardiol Pol. 2020; 78: 124-130.

2 Mollace V, Gliozzi M. The potential role of TLR4/caveolin-1/NOS pathway in oxyLDL-modulation of autophagic/apoptotic responses in endothelial cells. Int J Cardiol. 2016; 203: 457-458.

3 Mollace V, Gliozzi M, Musolino V, et al. Oxidized LDL attenuates protective autophagy and induces apoptotic cell death of endothelial cells: role of oxidative stress and LOX-1 receptor expression. Int J Cardiol. 2015; 184: 152-158.

4 Mollace V, Muscoli C, Masini E, et al. Modulation of prostaglandin biosynthesis by nitric oxide and nitric oxide donors. Pharmacol Rev. 2005; 57: 217-252.

5 Salvemini D, Kim SF, Mollace V. Reciprocal regulation of the nitric oxide and cyclooxygenase pathway in pathophysiology: relevance and clinical implications. Am J Physiol Regul Integr Comp Physiol. 2013; 304: R473-R487.

6 Frank PG, Galbiati F, Volonte D, et al. Influence of caveolin-1 on cellular cholesterol efflux mediated by high-density lipoproteins. Am J Physiol Cell Physiol. 2001; 280: C1204-C1214.

7 Feron 0, Belhassen L, Kobzik L, et al. Endothelial nitric oxide synthase targeting to caveolae. Specific interactions with caveolin isoforms in cardiac myocytes and endothelial cells. J Biol Chem. 1996; 271: 22810-22814.

8 Michel JB, Feron 0, Sacks D, Michel T. Reciprocal regulation of endothelial nitric-oxide synthase by $\mathrm{Ca}^{2+}$-calmodulin and caveolin. J Biol Chem. 1997; 272: 15583-15586.

9 Feron 0 . Endothelial nitric oxide synthase expression and its functionality. Curr Opin Clin Nutr Metab Care. 1999; 2: 291-296.

10 Sun SW, Zu XY, Tuo QH, et al. Caveolae and caveolin-1 mediate endocytosis and transcytosis of oxidized low density lipoprotein in endothelial cells. Acta Pharmacol Sin. 2010; 31: 1336-1342. 